\title{
A Compact Tunable Coherent Terahertz Source based on an Hybrid Integrated Optical Phase-lock Loop
} \author{
M. J. Robertson ${ }^{2}$, L. Pavlovic ${ }^{3}$, L. Naglic ${ }^{3}$, M. Vidmar ${ }^{3}$ and A. J. Seeds ${ }^{1}$ \\ ${ }^{1}$ Department of Electronic and Electrical Engineering \\ University College London \\ Torrington Place, London WC1E 7JE, United Kingdom \\ a.seeds@ee.ucl.ac.uk \\ ${ }^{2}$ CIP Technologies Ltd. \\ Adastral Park, Ipswich, IP5 3RE, United Kingdom \\ ${ }^{3}$ Faculty of Electrical Engineering \\ University of Ljubljana \\ 1000 Ljubljana, Slovenia
}

L. Ponnampalam ${ }^{1}$, R. J. Steed ${ }^{1}$, M. J. Fice ${ }^{1}$, C. C. Renaud ${ }^{1}$, D. C. Rogers ${ }^{2}$, D. G. Moodie ${ }^{2}$, G. D. Maxwell ${ }^{2}$, I. F. Lealman ${ }^{2}$,

\begin{abstract}
A tuneable terahertz source based on the first hybrid integrated optical phase-lock loop is presented. Generated signals have linewidth $<1 \mathrm{kHz}$ and phase noise $<-80 \mathrm{dBc} / \mathrm{Hz}$ at $10 \mathrm{kHz}$ offset. The measured output power at $300 \mathrm{GHz}$ was $-22 \mathrm{dBm}$.
\end{abstract}

\section{INTRODUCTION}

There has been an increasing interest in the development of cost effective, small footprint, low power consumption, coherent terahertz $(\mathrm{THz})$ sources $(100 \mathrm{GHz}-10 \mathrm{THz})$ for applications such as security/bio-medical imaging, high speed short reach wireless communications and instrumentation. In this paper we present a system, based on photonic mixing of two locked lasers in a fast photodetector [1-2], which is capable of generating widely tuneable signals with high spectral purity (linewidth $<1 \mathrm{~Hz}$ ) and high $\mathrm{CW}$ output power [3]. To achieve high spectral purity in such an heterodyne system, the outputs of the two slave lasers are phase locked to two different comb lines of an optical frequency comb generator (OFCG : master laser) using optical phase-lock loops (OPLL) (Fig. 1). This technique is also suitable for integration as described in this paper, making a compact, cost effective $\mathrm{THz}$ system realizable. In order to be able to lock slave lasers with Lorentzian linewidths of $1 \mathrm{MHz}-2 \mathrm{MHz}$ (full width half maximum: FWHM), typical of distributed Bragg reflector (DBR) lasers, it is necessary to achieve short loopdelay. The OPLL in this work employs an hybrid integration platform to integrate the InP based optical components on to a silica on silicon motherboard [4], reducing the optical delay to less than 50ps. With a custom-designed short delay electronic loop filter we have achieved the necessary short delay to lock a $1 \mathrm{MHz}$ linewidth slave laser to the comb lines of an OFCG.

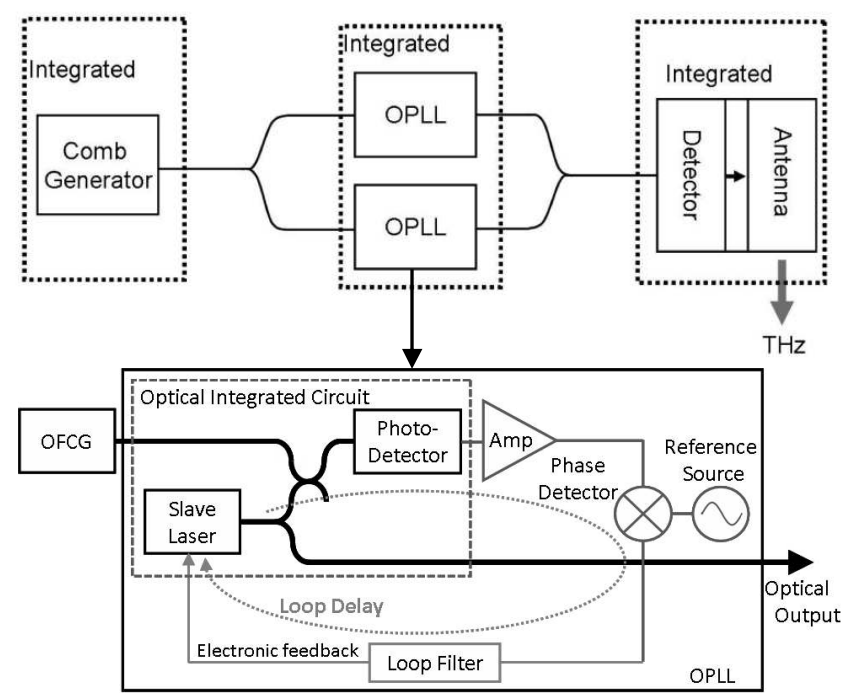

Figure 1. Schematic of a tuneable coherent $\mathrm{THz}$ source.

In an OPLL system, the frequency of the slave laser can be offset from the master comb line by an arbitrary value by tuning the frequency of the reference source [5]. However, the achievable offset frequency range is limited by the bandwidth of the photodetector and the components used in the shortdelay electronic loop filter circuits. In this paper we describe the performance of the OPLL over the offset locking range, 
which is critical for generation of continuously tunable coherent heterodyne signals.

Section II discusses the design and performance of the hybrid integrated OPLL, while Section III presents results for the $\mathrm{THz}$ synthesis system that uses the integrated OPLL to generate signals up to $300 \mathrm{GHz}$. The architecture demonstrated has the potential to generate signals up to $1.8 \mathrm{THz}$.

\section{HYBRID INTEGRATED OPLL}

\section{A. Design}

In an OPLL system, the heterodyne signal between the master and the slave lasers is amplified and compared with a microwave reference signal in a phase detector (Fig. 1). The error signal generated at the phase detector output is processed by the loop filter and fed back to tune the slave laser to achieve phase-lock between the master and the slave lasers. Only the phase variations of the master and slave lasers within the loop bandwidth will be compensated. The loop bandwidth is determined by the loop gain, loop filter response, frequency responses of the components and the loop delay. However, the loop delay places the fundamental limit on the loop bandwidth, as it is necessary for the feedback to be fast enough to track the phase variations associated with wide linewidth lasers. Hence it is necessary to minimize the delay associated with both the optical and electrical paths.

In this work, the delay requirements are quantified by calculating the tolerable maximum summed linewidth (master + slave laser linewidths) with loop delay, assuming that the phase variance is less than $0.03 \mathrm{rad}^{2}$ so as to ensure a mean time between cycle slips of several decades [6-7]. To lock master and slave lasers with summed linewidths of $2 \mathrm{MHz}$, this calculation yielded a loop delay of 1.6ns as the upper limit for a first order filter, and 1ns for a second order type II filter with a damping factor of 0.707 .

To minimize the optical delay, the InP based slave lasers and the photodetectors are hybrid integrated on to a silicon motherboard. The motherboard was designed such that the distance between the slave laser and the photodetector was less than $10 \mathrm{~mm}$ to give an optical path delay of less than $50 \mathrm{ps}$, allocating the larger fraction of the overall delay for the electrical path. In the hybrid integration platform, the performance of each of the optical components are optimized and fabricated separately and flip-chip bonded on to silicon daughter boards containing electrical connections. These are then flip-chip bonded on to the motherboard that contains the silica waveguide, electrical connections and coplanar waveguides (Fig. 2)

The electronic loop filter in this work was designed to include an integrator to achieve a wider tracking range, giving a second order loop response. In order to achieve a delay of less than $1 \mathrm{~ns}$ in the electronic path, the proportional (P-loop) and the integral (I-loop) paths are separated. This allows the proportional path to be kept simple with $3 \mathrm{~dB}$ bandwidth exceeding $1 \mathrm{GHz}$ and total time-delay of less than $1 \mathrm{~ns}$, while the much slower integral path ensures that slow frequency drifts are tracked over a wide frequency range. The OPLL electronics was implemented using commercial $10 \mathrm{~Gb} / \mathrm{s}$ emitter coupled logic and current mode logic integrated circuits, with phase detection carried out in XOR gates [8]. Due to the frequency response of various components in the electronic circuits, the offset frequency range is limited to 2 $7 \mathrm{GHz}$. The slow integral loop also includes a saw-tooth sweep generator for initial acquisition. The electronic circuit is built on a four layer FR-4 printed circuit board (PCB), connected to the hybrid OPLL motherboard by wire bonds.

Fig. 2 shows a picture of the dual OPLL system which consists of a motherboard mounted on a Peltier cooler and the two PCBs. The motherboard was designed to host a monolithically integrated pair of DBR lasers and two photodetectors. The twin DBR lasers are buried heterostructure lasers on a single $\mathrm{InP} / \mathrm{InGaAsP}$ chip, each laser having four sections : front and rear grating sections, gain section and phase section. The waveguide of each laser was of different width to achieve the necessary frequency offset between them. Each of the outputs of the lasers are further integrated with an angled tapered semiconductor optical amplifier (SOA) to boost the output power to $20 \mathrm{~mW}-25 \mathrm{~mW}$ with minimum facet reflections. The SOAs are operated at saturation to minimize the noise due to amplified spontaneous emission. The output from each of the lasers had SMSR greater than $40 \mathrm{~dB}$ and a tuning range of $7-8 \mathrm{~nm}$ with an offset of $6 \mathrm{~nm}$ between them at zero tuning currents, giving an heterodyne tuning range of $0 \mathrm{~Hz}$ to $1.8 \mathrm{THz}$ (Fig.3).

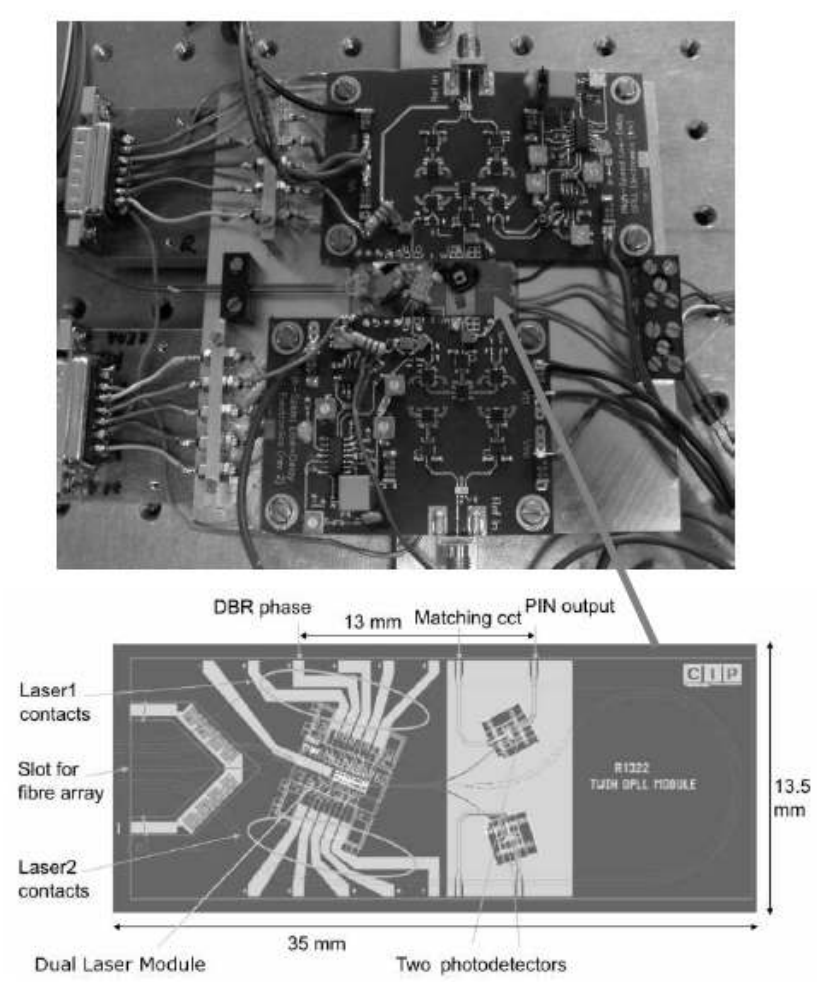

Figure 2. Picture of the complete double OPLL system with dimension of $15 \mathrm{~cm} \times 11 \mathrm{~cm}$, including the PCBs, and the layout of the motherboard itself. 


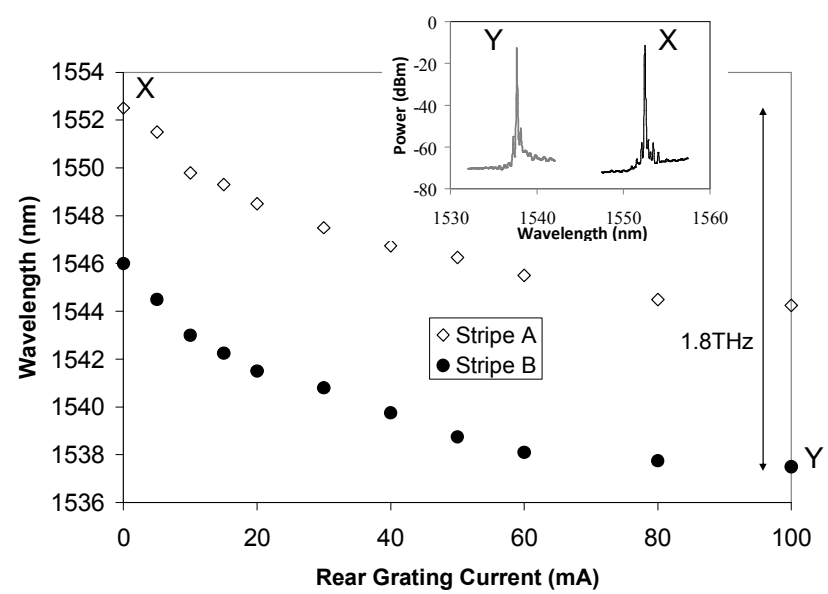

Figure 3. Wavelength tuning range of each of the stripes in the monolithic twin DBR lasers (Inset : Optical spectrum of the twin lasers with and without the tuning currents).

The linewidths of the DBR lasers were measured to be around $20 \mathrm{MHz}$ using self-heterodyne techniques. This is typical of any DBR laser where the measured linewidth is dominated by the low-frequency $1 /$ f noise component which has a Gaussian distribution [9]. However, the loop bandwidth is sufficient to compensate for the low frequency phase noise and hence only the Lorentzian linewidth is critical to the performance of the OPLL system. The Lorentzian linewidth of these lasers was measured to be $1 \mathrm{MHz}$, requiring the maximum allowable loop delay to be $1 \mathrm{~ns}$.

Large spot size InP electroabsorption modulators (EAMs) with angled facets are used as the photodetectors on the motherboard. The angled facets are anti-reflection coated to minimize back reflection into the lasers. The whole photonic integrated circuit (PIC) board was designed to have back reflection less than $-50 \mathrm{~dB}$ which was necessary to prevent any disruption in performance. These photodetectors have a $3 \mathrm{~dB}$ bandwidth of $10 \mathrm{GHz}$ and responsivity of $0.5 \mathrm{~A} / \mathrm{W}$ before integration. A coplanar waveguide was used to carry the RF signal from the detector to the PCB.

\section{B. Performance}

The OPLL performance was assessed by monitoring the heterodyne signal between the output of the OPLL and the master laser using an external fast photodiode and an RF spectrum analyzer. An external cavity tunable laser was used as the master laser, which had a FWHM linewidth of $100 \mathrm{kHz}$. The minimum RF power level required by the loop electronics is $-40 \mathrm{dBm}$ to $-25 \mathrm{dBm}$, depending on the offset frequency. This corresponds to a photocurrent level of $64 \mu \mathrm{A}$ to $356 \mu \mathrm{A}$ at the detector in the PIC. Hence the slave laser was operated with $150 \mathrm{~mA}$ into each of the gain and the SOA sections, and the master laser power was set to give $7 \mathrm{dBm}$ at the detector, to provide $-28 \mathrm{dBm}$ at the output of the detector. Phaselocking was achieved by slowly tuning the slave laser frequency such that the heterodyne signal frequency becomes closer to the microwave reference signal frequency.

Fig. 4 shows the spectrum of an unlocked signal, and the locked signal when the offset frequency was set at $3 \mathrm{GHz}$,
$4 \mathrm{GHz}$ and $5 \mathrm{GHz}$. The linewidth of the un-locked signal was $70 \mathrm{MHz}$, predominantly due to frequency instability in both master and slave lasers, while that of the locked signal was less than $1 \mathrm{kHz}$, limited by the resolution bandwidth of the RF spectrum analyzer. The phase noise was measured to be less than $-80 \mathrm{dBc} / \mathrm{Hz}$ at an offset of $10 \mathrm{kHz}$, for a locking range of $3-5 \mathrm{GHz}$ (Fig. 5). However with an increased SOA current of $300 \mathrm{~mA}$, locking was achieved over the entire locking range of $2-7 \mathrm{GHz}$, with an optimum performance at $4 \mathrm{GHz}$ due to the frequency response of the loop filter electronics.

\section{GENERATION OF TERAHERTZ SIGNALS}

The generation of signals over a wider frequency range is accomplished by locking the slave lasers to different comb lines of an OFCG. Continuous tuning can be achieved with offset locking in OPLL systems as shown in Fig. 4. The photonic integrated circuit board in this work was designed to have two OPLLs for the generation of coherent $\mathrm{THz}$ signal. However, as only one of the OPLLs was operational in this first device, generation of $\mathrm{THz}$ signals was achieved by injection locking a DBR laser to another comb line to provide the other input to the fast photodiode. The OFCG used in this demonstration was an external cavity laser with phase modulation covering a frequency range of $300 \mathrm{GHz}$, and the linewidth of each of the comb lines was $100 \mathrm{kHz}$. The fast photodiode to generate the $\mathrm{THz}$ signal was an uni-travelling carrier (UTC) photodiode [10]. To assess the spectral purity of the synthesized signal, an UTC photodiode $(3 \mathrm{~dB}$ bandwidth of $110 \mathrm{GHz}$ and responsivity of $0.5 \mathrm{~A} / \mathrm{W}$ ) was used with a coplanar probe, external mixer and an RF spectrum analyzer for the frequency range up to $110 \mathrm{GHz}$ and the synthesized signal in this frequency range had a linewidth less than $1 \mathrm{kHz}$, limited by the resolution bandwidth of the spectrum analyzer.

Fig. 6 shows the generated power over the frequency range $140 \mathrm{GHz}$ to $300 \mathrm{GHz}$, using a photodiode with partially doped absorber layer with an integrated bow-tie antenna. The optical power at the detector was $15 \mathrm{dBm}$ and the photocurrent was $3 \mathrm{~mA}$. The power was measured using a calibrated large area Thomas Keating power meter. The frequency tuning was achieved by locking the OPLL to successive comb lines with an offset of $4 \mathrm{GHz}$ from each of the comb lines generated by the microwave reference source. The measured power output was $-22 \mathrm{dBm}$ at $300 \mathrm{GHz}$.

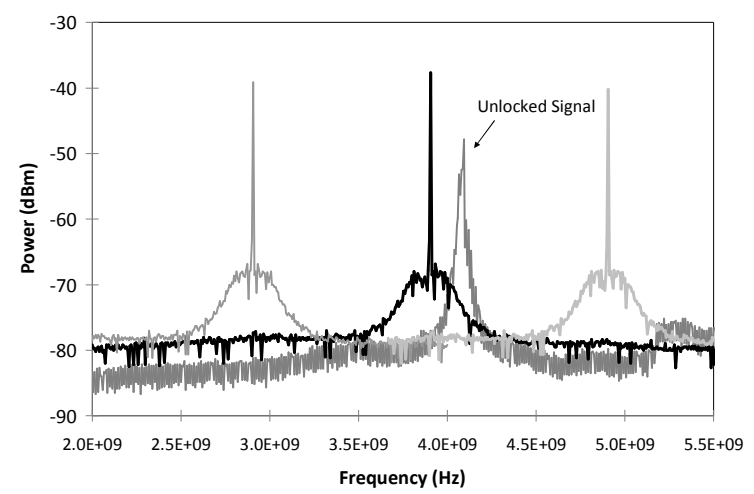

Figure 4. Spectrum of the unlocked signal at an offset of $4 \mathrm{GHz}$, and the locked signals at an offset of $3 \mathrm{GHz}, 4 \mathrm{GHz}$ and $5 \mathrm{GHz}$. 


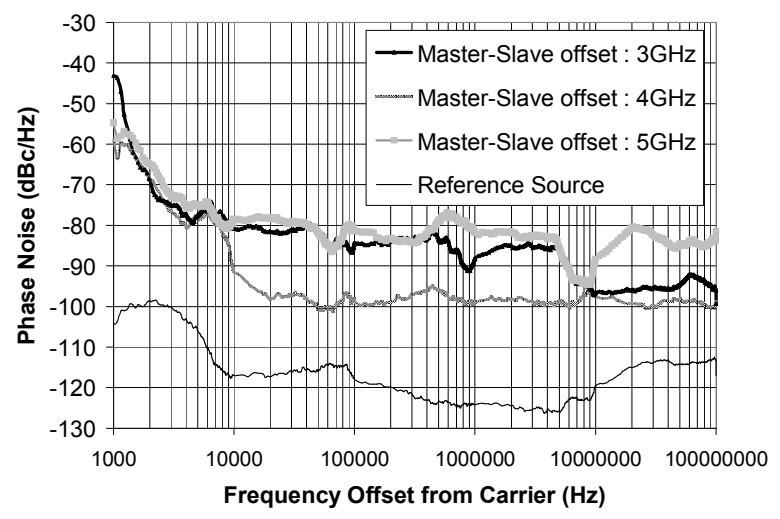

Figure 5. Phase noise measured for locking range of $3-5 \mathrm{GHz}$.

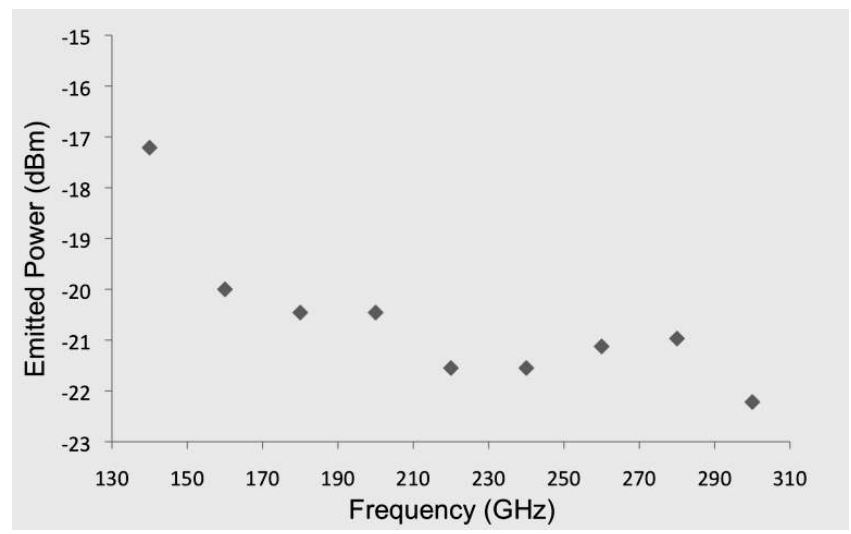

Figure 6. Power emitted from an antenna integrated photodiode measured by a calibrated $\mathrm{THz}$ power meter.

An OFCG with comb lines over a wider frequency range is required for the generation of signal beyond $1 \mathrm{THz}$, and the linewidth of each comb line has to be narrow $(<200 \mathrm{kHz})$ to achieve stable locking. A quantum dash modelocked semiconductor laser [11] having a comb linewidth of $70 \mathrm{kHz}$, with comb lines covering $1.6 \mathrm{THz}$ can be a viable solution, offering a compact source with a potential for integration with the OPLL PIC board. Moreover, by mounting the antenna integrated UTC photodetector on to a daughterboard and subsequently on to the same motherboard with a window to capture the radiated signal could lead to a $\mathrm{THz}$ source with similar dimensions to that of the integrated OPLL itself of $35 \mathrm{~mm} \times 14 \mathrm{~mm}$ (Fig. 2).

\section{CONCLUSIONS}

The first hybrid integrated OPLL system has been fabricated and tested. Phase locking to comb lines of an OFCG has been achieved with an offset locking range of $2 \mathrm{GHz}-7 \mathrm{GHz}$ by use of a microwave reference source. The key to this successful demonstration has been the low loop delay that was attained through hybrid integration and a custom designed fast electronic loop filter. The loop delay due to the optical path was $\sim 50 \mathrm{ps}$ while the electronic path delay has been estimated to be $\sim 1 \mathrm{~ns}$. The linewidth of the heterodyne signal between master and slaver lasers was measured to be less than $1 \mathrm{kHz}$, limited by the resolution bandwidth of the spectrum analyzer, and the phase noise was less than $-80 \mathrm{dBc} / \mathrm{Hz}$ at an offset frequency of $10 \mathrm{kHz}$.

Signals in the range of $10 \mathrm{GHz}$ to $300 \mathrm{GHz}$ have been synthesized using this OPLL PIC board, an injection locked DBR comb line filter and an OFCG. The measured power was $-3 \mathrm{dBm}$ at $100 \mathrm{GHz}$ and $-22 \mathrm{dBm}$ at $300 \mathrm{GHz}$ using UTC photodiodes

\section{ACKNOWLEDGMENT}

The authors would like to thank B. Harmon, P. Cannard, L. Rivers, R. Firth and P. Townley for their valuable assistance.

\section{REFERENCES}

[1] A. J. Seeds and K. J. Williams, "Microwave photonics," J. Lightw. Technol., vol. 24, pp. 4628-4641, 2006.

[2] Q. Quaraishi, M. Griebel, T. Kleine-Ostmann, and R. Braschitsch, "Generation of phase-locked and tunable continuous-wave radiation in the terahertz regime," Opt. Lett., vol. 30, no. 23, pp. 3231-3233, 2005.

[3] C. C. Renaud, M. Robertson, D. Rogers, R. Firth, P. Cannard, R. Moore, and A. J. Seeds, "A high responsivity, broadband waveguide uni-travelling carrier photodiode," in Proc. SPIE, vol. 6194, p.61940C, 2006.

[4] G. Maxwell, B. Manning, M. Nield, M. Harlow, C. Ford, M. Clements, S. Lucas, P. Townley, R. McDougall, S. Oliver, R. Cecil, L. Johnston, A. Poustie, R. Webb, I. Lealman, L. Rivers, J. King, S. Perrin, R. Moore, I. Reid, and D. Scrase, "Very low coupling loss, hybridintegrated all-optical regenerator with passive assembly," presented at the $28^{\text {th }}$ European Conference on Optical Communication, Copenhagen, Denmark, 2002.

[5] R. T. Ramos and A. J. Seeds, "Fast heterodyne optical phase-lock loop using double quantum well laser diodes," electron. Lett., vol. 28 , no. 1 , pp. 82-83, 1992.

[6] R.T. Ramos and A.J. Seeds, "Delay, linewidth and bandwidth limitations in optical phase-locked loop design", Electronic Letters, vol. 26, pp. 389, 1990.

[7] F.M. Gardner, Phaselock Techniques, Wiley-Blackwell, 2005.

[8] L. Naglic, L.Pavlovic, B.Bategelj and M. Vidmar, "Improved phase detector for electro-optical phase-locked loops", Electronic Letters, vol. 44 , no. 12,2008

[9] L. B. Mercer, "1/f frequency noise effects on self-heterodyne linewidth measurements," J. Lightw. Technol., vol. 9, no. 4, pp. 485-493, 1991.

[10] C.C. Renaud, L. Ponnampalam, F. Pozzi, E. Rouvalis, D. Moodie, M. Robertson and A.J. Seeds, "Photonically enabled communication systems beyond 1000GHz”, Microwave Photonics, pp.55-58, 2008.

[11] F. van Dijk, A. Enard, X. Buet, F.Lelarge and G.H. Duan, "Quantum dash mode-locked laser for millimeter-wave coupled opto-electronic oscillator", Microwave Photonics, IEEE International Topical Meeting on, pp.66-69, 2007. 